A reprint from

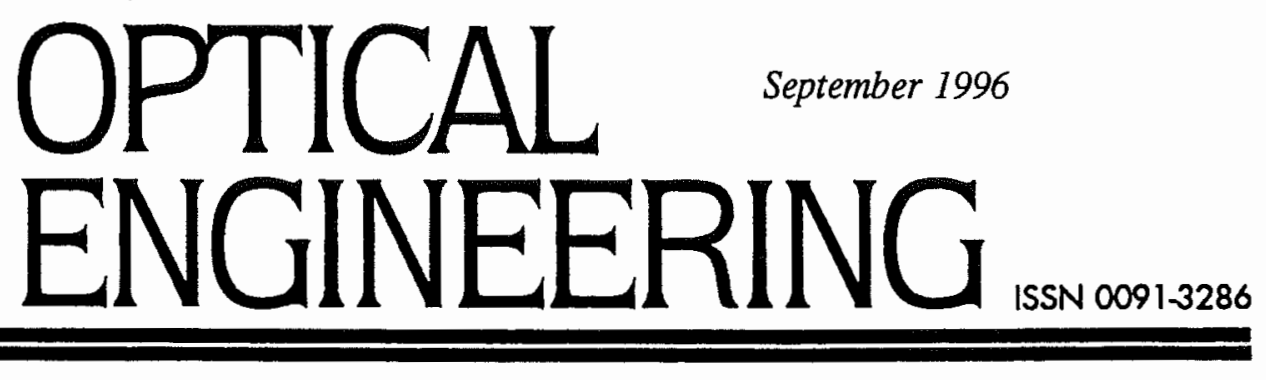

STRAIN MEASUREMENTS ON CONCRETE BEAM AND CARBON FIBER CABLE WITH DISTRIBUTED OPTICAL FIBER BRAGG GRATING SENSORS

Philipp M. Nellen

Rolf Brönnimann

Urs Sennhauser

Charles G. Askins

Martin A. Putnam 


\section{Strain measurements on concrete beam and carbon fiber cable with distributed optical fiber Bragg grating sensors}

\author{
Philipp M. Nellen \\ Rolf Brönnimann \\ Urs Sennhauser, MEMBER SPIE \\ EMPA \\ Swiss Federal Laboratories for Materials \\ Testing and Research \\ Section, Electronics/Metrology \\ Ueberlandstrasse 129 \\ $\mathrm{CH}-8600$ Dübendorf, Switzerland \\ E-mail: nellen@empa.ch \\ Charles G. Askins \\ Martin A. Putnam \\ Naval Research Laboratory \\ Code 5612 \\ 4555 Overlook Avenue Southwest \\ Washington, D.C. 20375-5000
}

\begin{abstract}
We report on civil engineering applications of wavelength multiplexed optical fiber Bragg grating arrays directly produced on the draw tower for testing and surveying advanced structures and materials such as carbon fiber reinforced concrete elements and prestressing cables. We equipped a $6 \times 0.9 \times 0.5 \mathrm{~m}$ concrete beam, which was reinforced with carbon fiber reinforced epoxy laminates, and a 7-m long prestressing carbon fiber cable made of seven twisted strands, with optical fiber Bragg grating sensors. Static strains up to $8000 \mu \mathrm{m} / \mathrm{m}$ and dynamic strains up to $1200 \mu \mathrm{m} / \mathrm{m}$ were measured with a Michelson interferometer used as Fourier spectrometer with a resolution of about 10 $\mu \mathrm{m} / \mathrm{m}$ for all sensors. Comparative measurements with electrical resistance strain gauges were in good agreement with the fiber optic results. We installed the fiber sensors in two different arrangements: some Bragg grating array elements measured local strain while others were applied in an extensometric configuration to measure moderate strain over a base length of 0.1 to $1 \mathrm{~m}$. (c) 1996 Society of Photo-Optical Instrumentation Engineers.
\end{abstract}

Subject terms: civil engineering; strain measurements; fiber optic sensors; Bragg gratings.

Paper 02125 received Dec. 1, 1995; revised manuscript received Feb. 28, 1996; accepted for publication Feb. 29, 1996. This paper is a revision of a paper presented at the SPIE conference on Distributed and Multiplexed Fiber Optic Sensors V, June 1995, Munich, Federal Republic of Germany. The paper presented there appears (unrefereed) in SPIE Proceedings Vol. 2507.

\section{Introduction}

Absolute strain measurements with fiber optic distributed sensors are of main interest for testing and surveying advanced structures in civil engineering. ${ }^{1-5}$ In particular, new components such as glass fiber reinforced ground anchors, carbon fiber reinforced concrete elements and prestressing cables in structurally important locations have to be monitored over their lifetime.

Optical fiber Bragg grating (BG) arrays are well suited for distributed strain sensors either fastened to the surface or embedded in the material. In particular for distributed sensing purposes and for measurements of strain inside materials (embedded), they provide solutions that are difficult or even impossible to realize with conventional electrical resistance strain gauges.

A uniform fiber optic Bragg grating with a grating period of $\Lambda$ and a mean refractive index of $n$ reflects at a Bragg wavelength of $\lambda_{B}{ }^{6}$

$\lambda_{\mathrm{B}}=2 n \Lambda$.

If the applied strain change $\Delta \epsilon$ is uniform over the grating length, the Bragg wavelength change $\Delta \lambda_{B}$ at constant temperature is ${ }^{7}$ :

$\Delta \lambda_{\mathrm{B}} / \lambda_{\mathrm{B}}=\left(1-p_{e}\right) \Delta \epsilon$
Here $p_{e}=0.204$ is the effective elasto-optic coefficient. ${ }^{8}$ Equation (2) is only valid for negligible transverse loading, or loading that resembles Poisson contraction and expansion. Bragg gratings also respond to temperature changes $\Delta T:$

$\Delta \lambda_{\mathrm{B}} / \lambda_{\mathrm{B}}=(\alpha+\xi) \Delta T$,

with $\alpha=0.55 \times 10^{-6} / \mathrm{K}$ (thermoelastic coefficient) and $\xi=8.3 \times 10^{-6} / \mathrm{K}$ (thermo-optic coefficient).

\section{Experiment}

\subsection{Production and Characterization of Optical Fiber Bragg Grating Arrays}

The arrays of wavelength-stepped Bragg gratings used in this work were written into the fiber with a single laser pulse $^{9}$ as it was fabricated on an otherwise conventional draw tower. Immediately before the protective polymer coating was applied to the freshly drawn fiber, it passed through the intersection region of a writing interferometer where a $\sim 10$ ns pulse from a krypton-fluoride excimer laser generated the periodic index modulation in the fiber core. A wavelength-addressable array was produced by a computer-controlled adjustment of the interferometer's geometry after each pulse so that the desired sequence of 


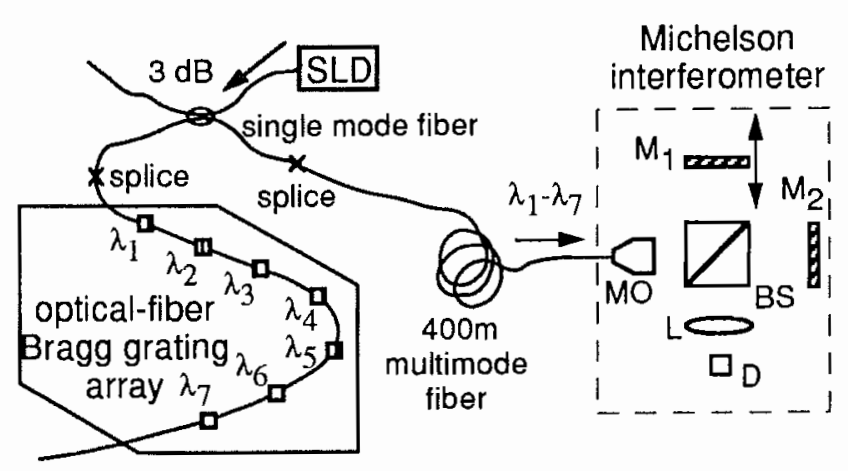

Fig. 1 Optical setup. Computer-controlled remote Michelson interferometer and optical fiber $B G$ array in test specimen. $M_{1}$, movable mirror; $M_{2}$, fixed mirror; $B S$, beamsplitter; $M O$, microscope objective; L, lens; $D$, silicon photodetector; and SLD, superluminescence diode.

Bragg wavelengths resulted. The distances between the elements were determined by properly setting the repetition rate of the laser.

Each seven-element array in this report was formed in approximately $5 \mathrm{~s}$, which included writing, coating, curing, and marking of the elements. The core of the silica host fiber had a germanium content of approximately 15 molar percent, a core diameter of $\sim 3.5 \mu \mathrm{m}$, a second-mode cutoff wavelength of $\leqslant 780 \mathrm{~nm}$, an outside diameter near $82 \mu \mathrm{m}$, and a UV-cured acrylate buffer coating of $\sim 180 \mu \mathrm{m}$ diameter. Each grating element in the array was approximately $6 \mathrm{~mm}$ in length, and the 2 to $3 \%$ reflectivities and $\sim 0.04$ $\mathrm{nm}$ bandwidths were determined with a scanning Ti:sapphire laser referenced to a calibrated Fabry-Perot interferometer. ${ }^{10}$ The presence of gratings within the fiber did not affect its breaking strength, which in all cases exceeded $700 \mathrm{kpsi}$ (4.8 GPa).

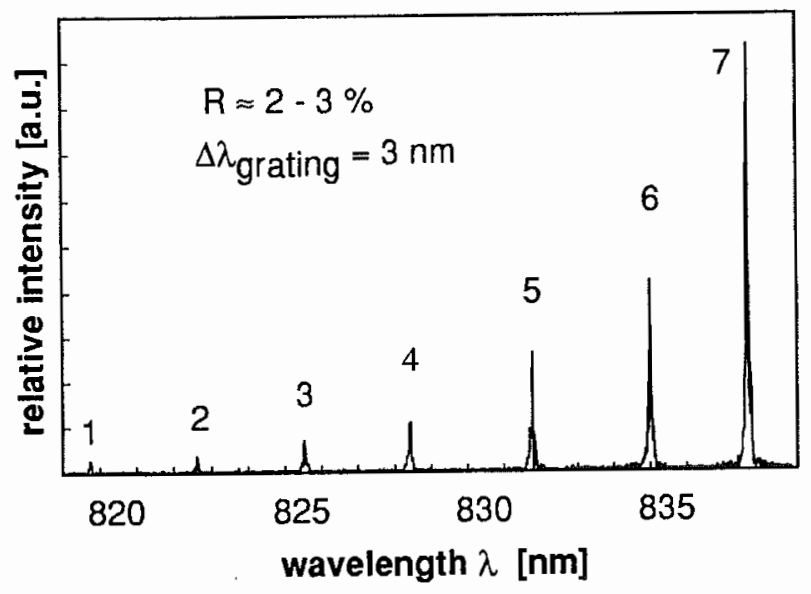

Fig. 2 Representative reflection spectrum of optical fiber Bragg grating array illuminated with a superluminescence diode and measured with a Michelson interferometer. Reflectivities are 2 to $3 \%$, reflection bandwidths are approximately $0.04 \mathrm{~nm}$ FWHM, and the spectral spacing between BGs is $3 \mathrm{~nm}$.

\subsection{Experimental Setup and Data Analysis}

A schematic drawing of the optical system is shown in Fig. 1. The light of a superluminescence diode (SLD, $\lambda=$ $850 \mathrm{~nm}, \Delta \lambda=24 \mathrm{~nm}, P \approx 114 \mu \mathrm{W}$ in the fiber) is coupled into one arm of a fiber optic $3-\mathrm{dB}$ coupler and passes to the optical fiber Bragg grating array. No special care was taken to adjust the polarization. Only light corresponding to the individual Bragg wavelengths of the seven gratings is reflected back and coupled into a multimode fiber (core/ cladding: $50 \mu \mathrm{m} / 125 \mu \mathrm{m}$ ). The $400-\mathrm{m}$ long multimode fiber guides the signals of the sensors to a computercontrolled scanning Michelson interferometer (MI). This remote sensing arrangement was used because the tests were performed in a large testing hall and the experimental MI was built for use in the laboratory.

An MI scan was performed in about $1 \mathrm{~min}$ with a velocity of $0.5 \mathrm{~mm} / \mathrm{s}$ and a scanning step of $261 \mathrm{~nm}$ (about one-third of the typical Bragg wavelength of $\lambda_{\mathrm{B}}=830 \mathrm{~nm}$ ). Taking $2^{17}$ samples per scan gives an optical sampling path of $34.2 \mathrm{~mm}$, or a step of $\Delta k=0.29 \mathrm{~cm}^{-1}$ in wave number $\bar{k} \equiv 1 / \lambda$. This corresponds to a step in wavelength $\lambda$ of $\Delta \lambda=\Delta k / k^{2}=0.02 \mathrm{~nm}$ or to a change in strain $\epsilon$ of $\Delta \epsilon=$ $30 \mu \mathrm{m} / \mathrm{m}$ [Eq. (2)].

The Bragg wavelengths $\lambda_{\mathrm{B}}$ of the strained gratings in the array were measured simultaneously, i.e., the gratings were multiplexed in series. The raw data of one MI scan were composed of cosinelike functions, one for each Bragg reflection (see Fig. 1 for optical setup). The power spectrum of the back-reflected light was obtained (Fig. 2) from a Fourier transform (FFT) of the interferogram of an MI scan. The Bragg wavelengths $\lambda_{B}$ were determined at the center of gravity for each of the seven reflection peaks. We obtained an experimental reproducibility in Bragg wavelength $\lambda_{\mathrm{B}}$ of $\Delta \lambda_{\mathrm{B}}=0.002 \mathrm{~nm}$ for a signal-to-noise ratio (SNR) of 60:1 and of $\Delta \lambda_{\mathrm{B}}=0.01 \mathrm{~nm}$ for an SNR of 3:1 (worst case) corresponding to strain uncertaintics of $\Delta \epsilon=$ $3 \mu \mathrm{m} / \mathrm{m}$ and $\Delta \epsilon=15 \mu \mathrm{m} / \mathrm{m}$, respectively.

The sensor bandwidth and signal-to-noise ratio reported here is not a basic limitation of strain detection with fiber BG arrays. A prototype instrument based on a grating spectrometer has demonstrated less than $3 \mu \mathrm{m} / \mathrm{m}$ noise at $20-\mathrm{Hz}$ scan rates with the same grating reflectivities and launched optical power. ${ }^{11}$ Here, to achieve a good SNR with conventional sources and low-reflectivity gratings, the reflected signals from all BGs were simultaneously integrated over the entire measurement period by a linear $\mathrm{CCD}$ imager.

\subsection{Installation of Optical Fiber Bragg Grating Arrays}

\subsubsection{Concrete beam}

We equipped a $6 \times 0.9 \times 0.5-\mathrm{m}$ concrete beam (T-shape, resonance frequency about $20 \mathrm{~Hz}$ ) with an optical fiber Bragg grating array (see Fig. 3). The mechanical properties of the beam were investigated when it was reinforced with carbon fiber reinforced epoxy laminates on the whole area of its bottom side. ${ }^{12}$ The concrete beam was symmetrically supported with steel bearings. In between two points, loads of up to $F_{\max }=283.4 \mathrm{kN}$ could be introduced statically and dynamically at low frequencies (four-point bending). The 

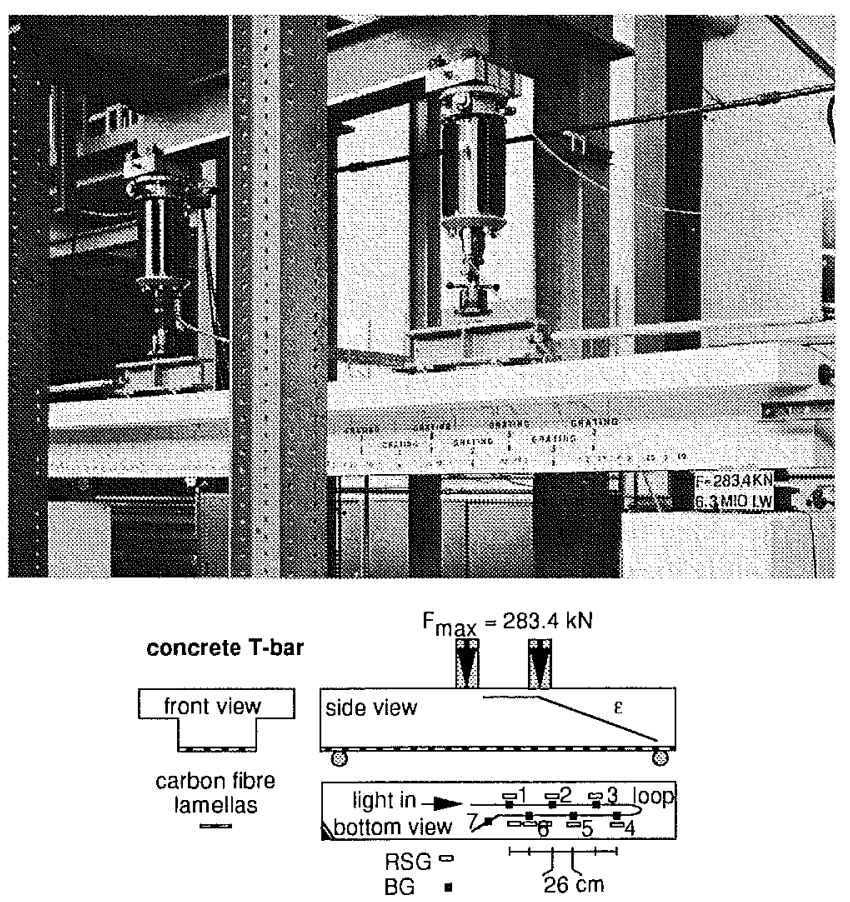

Fig. 3 Photography and schematic view of the concrete beam reinforced with carbon fiber reinforced epoxy laminates (dimensions of beam $6 \times 0.9 \times 0.5 \mathrm{~m}$ ). Shown are the positions and the numbering of the sensor Bragg gratings (BG) and the corresponding electrical resistance strain gauges (RSG) (BG length, $6 \mathrm{~mm}$; RSG length, $10 \mathrm{~mm}$ ).

concrete beam had already experienced $6.3 \times 10^{6}$ load cycles and showed several cracks when we started our measurements.

A seven-element BG array was attached to the laminates (Fig. 3). By backfolding the fiber between gratings 3 and 4, a sensor distribution with a spacing of $26 \mathrm{~cm}$ over a range of $160 \mathrm{~cm}$ was obtained. Gratings 1 and 6 were positioned between the two load introduction points, a region of expected constant strain; gratings 2 to 5 were positioned in a region with a strain gradient. In the first experiments, grating 7 was loosely mounted to monitor the influence of temperature. Later it was configured as a fiber extensometer by fixing two $10-\mathrm{cm}$-long regions of the fiber to the laminates and leaving a $1-\mathrm{m}$-long segment containing the grating freely slipping in between. To attach the optical fiber with the BGs to the carbon fiber reinforced epoxy laminates, the fiber was slightly prestressed. Glue was painted on with a thin brush along the fiber. The vacuum bagging method was used to press the fiber homogeneously to the laminates and to absorb the residue of the glue. After $24 \mathrm{~h}$ the vacuum bag was removed. Resistance strain gauges (RSGs) were then mounted beside the gratings. Two additional RSGs were attached at either side of grating 6 at a distance of $1.5 \mathrm{~cm}$ along the fiber to gather information about strain inhomogeneities.

\subsubsection{Prestressing carbon fiber cable}

Prestressing cables made of carbon fibers are currently under investigation as substitutes for steel cables, e.g., in prestressed concrete bridges. In contrast to steel, which exhibits considerable plastic deformation after its yield strength carbon fiber cable

- extensometric BG r local BG
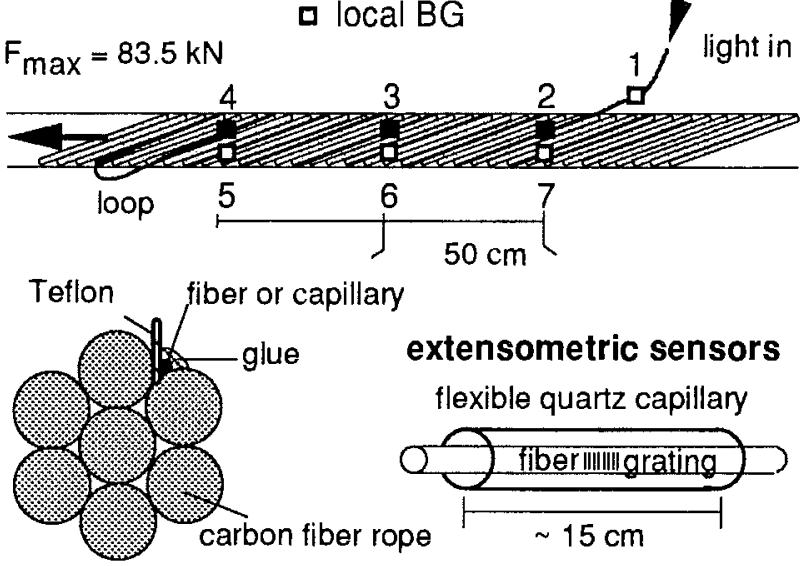

Fig. 4 Schematic view of the carbon fiber prestressing cable. The arrangement of the local point and extensometric Bragg gratings is shown. A cross-sectional drawing of the cable with details of fiber installation and a schematic view of an extensometric sensor is added.

has been reached, carbon fiber material is perfectly elastic up to rupture. Therefore, it is important to closely monitor the strain of carbon fiber cables. We investigated the application of $\mathrm{BGs}$ to a $7-\mathrm{m}$-long prestressing cable consisting of seven twisted carbon fiber strands with a lay length of $15 \mathrm{~cm}$ and a cross-section of about $76 \mathrm{~mm}^{2}$ (Fig. 4). Each strand was covered separately with a protective web to prevent damage.

The fiber was placed in the slit between two strands and was wound spirally along the cable. To protect it against friction forces during pulling and against clamping by the two strands, a thin Teflon tape was inserted in parallel between two adjacent strands. The fiber could then be fixed to only one of two neighboring strands and was protected from slipping too deep into the slit during the hardening of the glue.

The seven BGs of the sensor array were installed in the prestressed carbon fiber cable as follows. BGs 5, 6, and 7 of an array were fixed at one strand in the middle of the cable and separated by $52 \mathrm{~cm}$ from each other. They were directly glued onto the protective web, measuring local strain at their position, i.e., they were local sensors. The fiber parts containing BGs 2,3 , and 4 were configured as extensometric sensors. They were first inserted into quartz capillaries, each of a length corresponding to the lay length of the cable, an inner diameter of $320 \mu \mathrm{m}$, and an outer diameter of $500 \mu \mathrm{m}$. Each capillary was spirally wound in the same way as the fiber and glued to one strand; the optical fiber was fixed at the ends and could slip inside the capillaries. The BGs in the middle of the capillaries therefore measured mean strain over one lay length and, in contrast to the local sensors, were insensitive to local strain inhomogeneities. Finally, BG 1 was left free to monitor the influence of temperature during the load cycles. 


\section{Results and Discussion}

\subsection{Static Strain Profile Measurements}

We characterized the spectral features of the gratings before and after the installation and for each step of a load cycle. The characterization included peak shape, height, width, and the determination of Bragg wavelength $\lambda_{\mathrm{B}}$. One load cycle consisted of several static loads increasing from zero up to the maximum load of $F_{\text {max }}$ and back (concrete beam: bending force $F_{\max }=283.4 \mathrm{kN}$, prestressing carbon fiber cable: pulling force $F_{\max }=F_{\text {break }} / 2=83.5 \mathrm{kN}$ ).

\subsubsection{Concrete beam}

The performance of the optical fiber Bragg grating array was tested by measuring the strain distribution and comparing it with resistance strain gauge measurements for several loads. A survey for one complete static load cycle is given in Fig. 5. The strains measured with all BGs for all loads were compared with the strains measured with the RSGs at the same positions [Fig. 5(a)]. The strains $\epsilon$ of all BGs were calculated from the measured Bragg wavelength shifts $\Delta \lambda_{\mathrm{B}}$ with no further assumptions [Eq. (2)]. Most measurements lay between the two lines corresponding to an error of $\pm(15 \mu \mathrm{m} / \mathrm{m}+0.02 \times \epsilon)$. This includes deviations not only due to the optical measurement system but also the electrical RSG measurement system and the slightly different position of BGs and RSGs.

The maximum Bragg wavelength shift of $\Delta \lambda_{\mathrm{B}}=0.79 \mathrm{~nm}$ for grating 6 , corresponds to a strain of $\epsilon=1200 \mu \mathrm{m} / \mathrm{m}$. The Bragg wavelength of reference grating 7 remained constant at $\lambda_{\mathrm{B}}=837.203 \mathrm{~nm}$. This means that compared with the measurement uncertainty of this grating of $\pm 0.005 \mathrm{~nm}$, the temperature in the testing hall changed less than $\Delta T<1{ }^{\circ} \mathrm{C}$ (Eq. 3). This result was also confirmed by an independent temperature measurement.

Figures 5(b) and 5(c) show the distributed strain field over a length of $1.6 \mathrm{~m}$ for the same load cycle as Fig. 5(a). The measurements made with BGs [Fig. 5(b)] are compared with those made with RSGs [Fig. 5(c)]. Cracks and material inhomogeneities are the reason that the measurements made with the three RSGs near grating 6 and in close vicinity to each other differ more than $\pm(5 \mu \mathrm{m} / \mathrm{m}+0.02$ $\times \epsilon)$, as would be expected in a homogeneous strain field. Thus, a comparison of strains measured with BGs and those measured with RSGs has to be done carefully. We conclude that the effective errors of strain measurements with BGs (compared with RSGs) are smaller than given here and depend more on the measurement conditions than on the measurement method.

\subsubsection{Prestressing carbon fiber cable}

Figure 6 shows the measured strain versus load for local sensors 6 and 7, for reference sensor 1 [Fig. 6(a)], and for extensometric sensors 2, 3, and 4 [Fig. 6(b)]. We found that for local sensors, the slopes varied three times more than for extensometric sensors. The different slopes may be the result of local shear forces on the twisted strands. From the slope of $(1.06 \pm 0.05) \times 10^{-2} \mathrm{kN} /(\mu \mathrm{m} / \mathrm{m})$ in the load-strain curve, the modulus of elasticity of the prestressing cable was calculated to be $E=(139 \pm 6) \mathrm{kN} \mathrm{mm}^{-2}$, which corresponds to the value of $140 \mathrm{kN} \mathrm{mm}^{-2}$ given by the manufacturer.
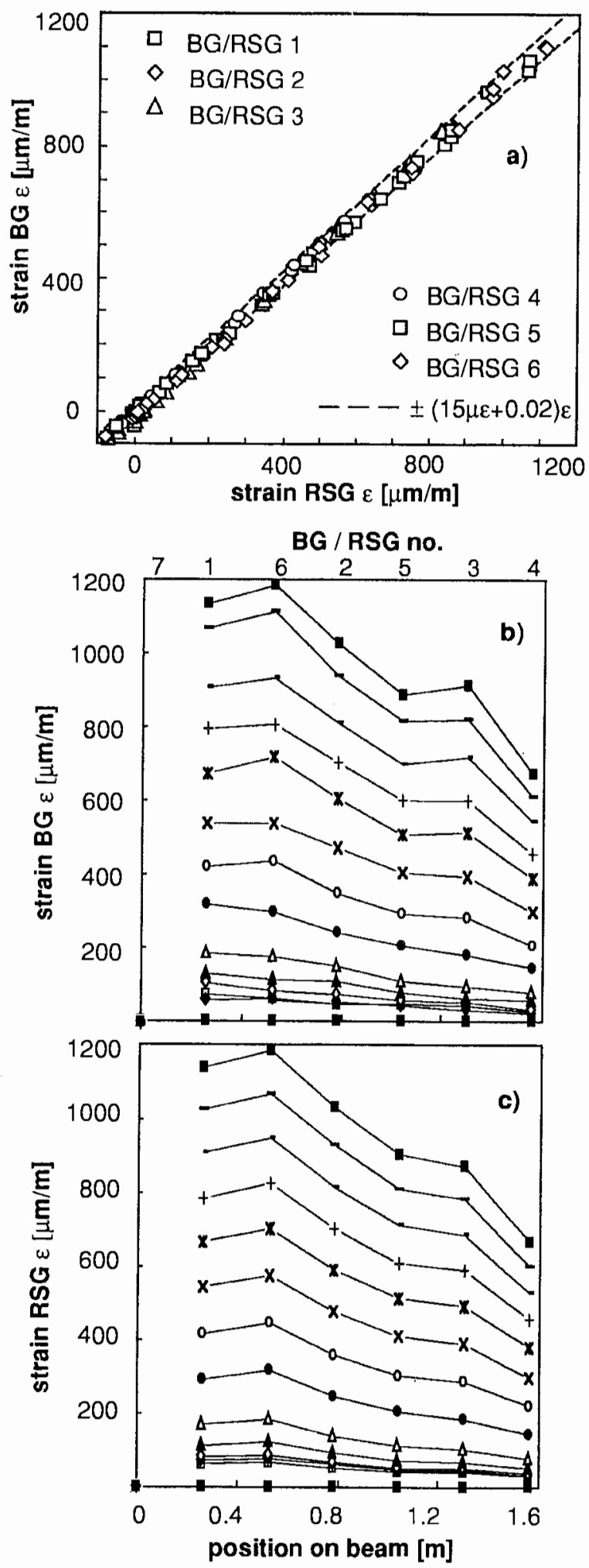

Fig. 5 Strain measurements on a concrete beam. The strain values $\epsilon$ measured with the different Bragg gratings $(B G, 1-6)$ at different loads are shown versus the value of the corresponding resistance strain gauges (RSG) at the same locations and loads (a). The dashed lines correspond to a deviation of $\pm(15 \mu \mathrm{m} / \mathrm{m}+0.02 \times \epsilon)$. The strain distributions over a length of $1.6 \mathrm{~m}$ of the beam are shown for increasing loads (different symbols) from $0,10,11,12$, $15,20,30, \ldots$ up to $100 \%$ of the maximum load measured (b) with optical fiber BGs (serially multiplexed with one optical fiber) and (c) with RSG (parallel multiplexed). 

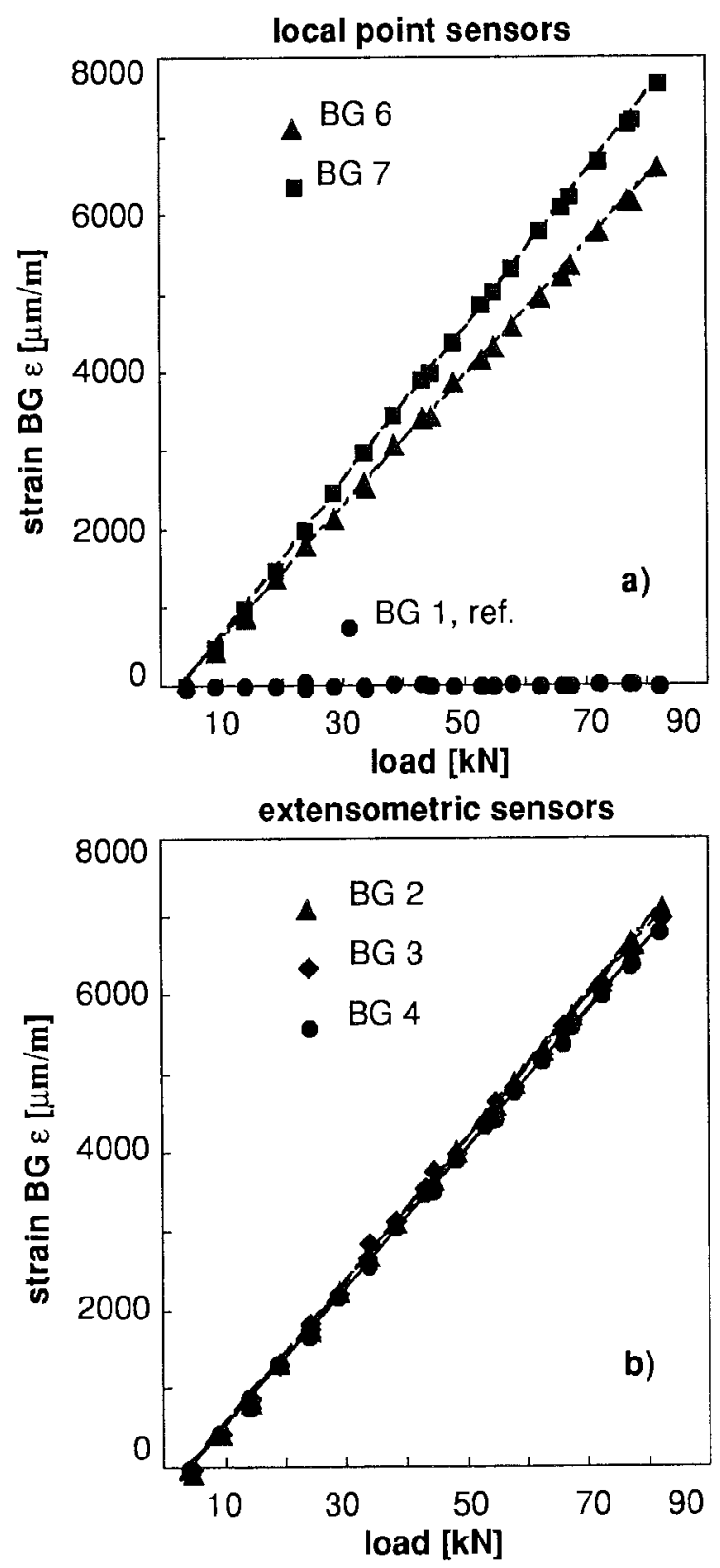

Fig. 6 Strain measurements on prestressing carbon fiber cable Strain versus load measured with (a) local point sensors and (b) extensometric Bragg grating sensors. BG sensors in an extensometric configuration show a better performance for strain measurements. Figure 6(a) contains measurements with the reference sensor.

We measured a slight nonlinearity (small quadratic term) in the elastic region of the load-strain curve of about $+(80 \pm 40) \mu \mathrm{N} /(\mu \mathrm{m} / \mathrm{m})^{2}$. This can be interpreted as an increase in the modulus of elasticity $E$ with strain, e.g., at a strain of $7500 \mu \mathrm{m} / \mathrm{m}$ the increase in $E$ is about $5 \%$ of the initial value. This is explained by the stranding, which caused the development of lateral confinement pressure with increasing tensile load, resulting in a measurable stiffening (i.e., an increase in the modulus of elasticity) of the cable. Such effects were also observed during other independent measurements. ${ }^{13}$
The special geometry of the cable would make it quite difficult to use RSGs for direct comparison. Therefore they were omitted. Such a prestressing cable is a typical object in which fiber optic sensors show their full advantage: the ability to measure the strain distribution with a single thin and flexible optical fiber, including the sensors embedded along one strand.

\subsubsection{Local point and extensometric Bragg grating sensors}

We took measurements at the shorter wavelength side of the maximum output power of the light source; therefore higher strains should result in higher peaks due to the increasing power source at longer wavelengths (see Fig. 2). Nevertheless, we observed a decreasing peak height with increasing strain and a corresponding broadening of the peak for some gratings.

In the case of the concrete beam, the peak sometimes split almost into two. This can be explained either by a nonuniform axial strain field or transverse stress that generated birefringence over the BG gauge length. In contrast to Ref. 14, debonding of the fiber from the laminates can be excluded because the peaks regained their original shape after the load was released, and the visual inspection of the glue line did not show any deterioration of the bond. Rather, we concluded that these inhomogeneities over the gauge length were caused by cracks in the vicinity of the BGs and RSGs, or by material inhomogeneities on or near the surface of the concrete.

To test the influence of local inhomogeneities, we installed grating 7 as a 1-m extensometer as described earlier. As a rule of thumb, the length of an RSG is normally chosen to be five to six times longer than the extension of the largest granulate in the concrete. A 1-m optical fiber extensometer clearly fulfills this criterion and the reflection peaks remained constant in their shape.

The degradation of the reflection peaks of local point sensors was also observed in the case of the prestressing carbon fiber cable. Especially at higher strains, the degradation was found to be more severe than in the case of the concrete beam. In Fig. 7 the changes in the reflection peak of a local point sensor [Fig. 7(a), with splitting and broadening] during a load cycle are compared with those of an extensometric sensor [Fig. 7(b), with nearly constant peak shape]. As an example, a peak in Fig. 7(a) broadened to a width of about $0.36 \mathrm{~nm}$ at the highest strain of $8000 \mu \mathrm{m} / \mathrm{m}$. This broadening, which was assumed to be caused by a nonuniform axial strain field, corresponded to a variation in strain of about $540 \mu \mathrm{m} / \mathrm{m}$ per $6 \mathrm{~mm}$ of the grating length or to a local gradient of $90,000 \mu \mathrm{m} / \mathrm{m}$ per meter. These inhomogeneities inside the BGs were introduced by the rough textile web protecting the strands; they were smeared out for the $15-\mathrm{cm}$-long extensometric sensors. In all extensometric measurements, the peak shape remained constant during the load cycles and showed a better accuracy and resolution than the local point measurements [see e.g., Fig. 6(b)].

\subsection{Strain Measurements on an Oscillating Concrete Beam}

We demonstrate that relevant information about the dynamic behavior of a concrete beam can be extracted from a 
point sensor
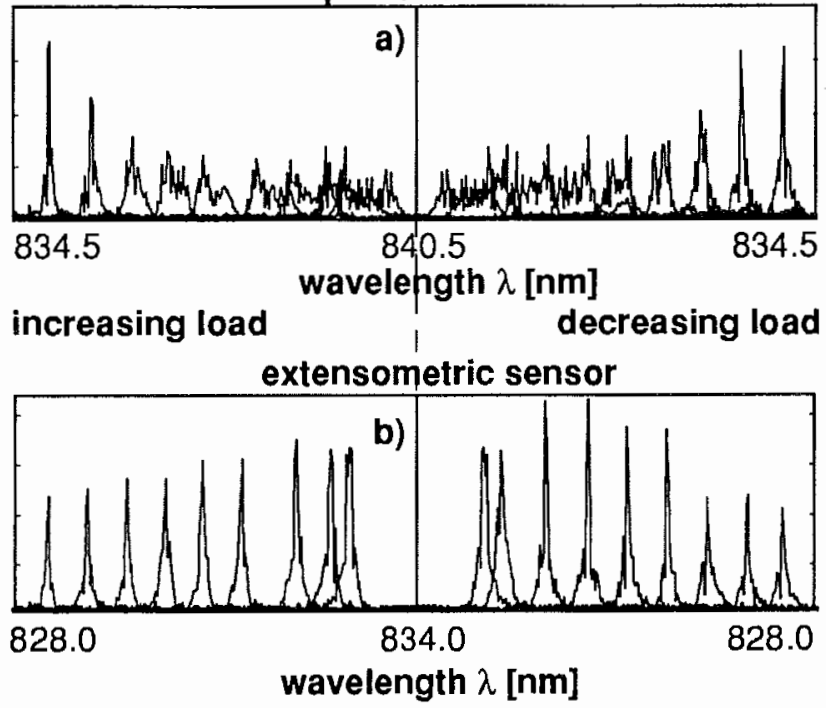

Fig. 7 Changes in reflection peak shape. The measurements were made on the prestressing carbon fiber cable. Peaks of local point BG sensors (a), e.g., grating 6 , broaden and show a multipeak shape at higher loads but regain their original shape with decreasing load. Peaks of BGs of extensometric configuration (b), e.g., grating 7 , remain sharp.

reflection interferogram of the $\mathrm{BG}$ array measured during the oscillation. This can be done although the MI is a rather slowly scanning instrument.

A periodic load $F(t)$ (mean load $F_{m}$ and amplitude $\Delta F_{a}$ ) exciting the concrete beam with a constant oscillation frequency $\Omega$ induces a periodic strain $\epsilon(t)$ and thereby a periodic Bragg wavelength change for each $B G$. From the reflection interferogram measured during the oscillation, the following parameters can be regained: frequency $\Omega$, mean value of the strain $\epsilon_{m}$, strain amplitude $\Delta \epsilon_{a}$, and strain $\epsilon_{\varphi}$ as a function of the oscillation phase angle $\varphi$.

If the MI scans an optical path $s$ with an (optical) velocity $v$ during continuous beam oscillation, the interferogram corresponds to the same phase angle $\varphi$ of the beam movement every $\Delta s=v / \Omega$ and therefore to the same momentary reflection spectrum of the BG array. The interferogram can be interpreted as being composed of partial interferograms containing only data points of a certain oscillation phase angle $\varphi$. Such a partial interferogram can be regarded as a product of the original interferogram and a periodic function that picks out the data every $\Delta s$. This periodicity of $\Delta s$ in $s$ leads to a periodicity in wave number $\bar{k}$ of $\Delta \bar{k}_{\Omega}=1 / \Delta s=\Omega / v$ of the spectrum because the Fourier transform of a product is the convolution of the Fourier transform of each factor.

Figure 8(a) shows the Fourier power spectrum calculated from a reflection interferogram that was measured during beam oscillation. From the measured $\Delta \bar{k}_{\Omega}$ and the known scanning velocity of the MI of $v=0.1 \mathrm{~m} \mathrm{~ms}^{-1}$, we obtained the applied excitation frequency $\Omega / 2 \pi=4.33 \mathrm{~s}^{-1}$. Since $\Delta \bar{k}_{\Omega}$ is proportional to $1 / v$, it can be adjusted to be larger than the overall spectral width of the seven BGs, i.e., the repeated Bragg reflection peaks of the seven gratings do not overlap.

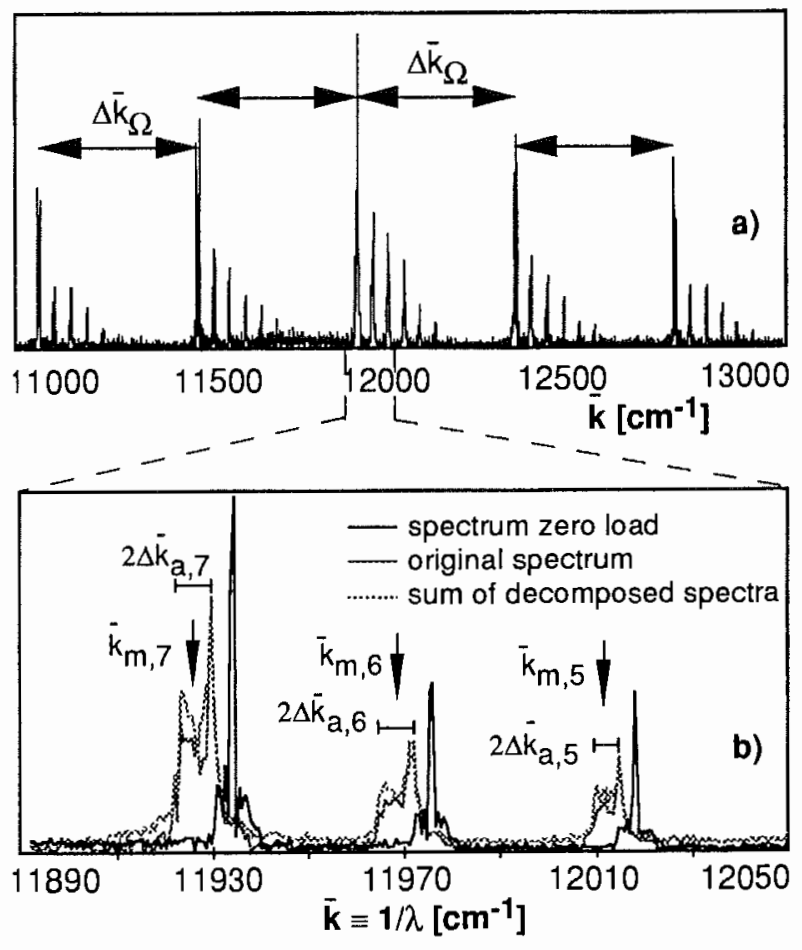

Fig. 8 (a) Fourier transform of interferogram measured during oscillation of the concrete beam; (b) detailed comparison of the reflection spectra of the unloaded beam and the oscillating beam. The spectrum for the zero load case is scaled by a factor of 0.4 for a better comparison. For the oscillating beam, the directly calculated spectrum is compared with the sum of the decomposed spectra.

In Fig. 8(b) the spectra for BGs 5 to 7 are given in more detail. The solid line with the sharp peaks is the measured reflection spectrum without load as reference. The dashed line shows the original spectrum measured during beam oscillation. From the center wave number $\bar{k}_{m}$ and the spectral width $2 \Delta \bar{k}_{a}$ of each reflection peak, the mean strain $\epsilon_{m}$ and the strain amplitude $\Delta \epsilon_{a}$ of the oscillation can be estimated. The dotted line shows the sum of the decomposed spectra [see Fig. 9(a) for explanation]. The difference is due to numerical approximations.

The reflection spectrum at this phase angle $\varphi$ is obtained by separately Fourier transforming the partial interferograms at a constant oscillation phase angle $\varphi$. In Fig. 9(a), the decomposed spectra for BG 7 are shown. The lowest trace corresponds to the unloaded concrete beam. The other traces correspond to the spectra of the decomposed interferogram measured during beam oscillation at phase angles of $\varphi=0$ to $360 \mathrm{deg}$ in steps of $30 \mathrm{deg}$. The spectra at phase angles 90 and $270 \mathrm{deg}$ are broadened and show smaller peak heights than the others because of a faster change of the spectral composition of the reflected light.

The Bragg wavelengths $\lambda_{\mathrm{B}}$ were evaluated with respect to phase angle $\varphi$. The results of BGs 2 to 7 converted to strain $\epsilon$ are shown in Fig. 9(b). All BGs show a sinusoidal dependence of strain $\epsilon_{\varphi}$ on phase angle $\varphi$. This finding does not depend on the assumption of a periodic oscillation, but is a result of the actual sinusoidal movement of the concrete beam. The mean strain $\epsilon_{m}$ and the strain amplitude $\Delta \epsilon_{a}$ for the BGs are shown in Fig. 9(c) and are compared with measurements made with RSGs. The ratio of load ampli- 

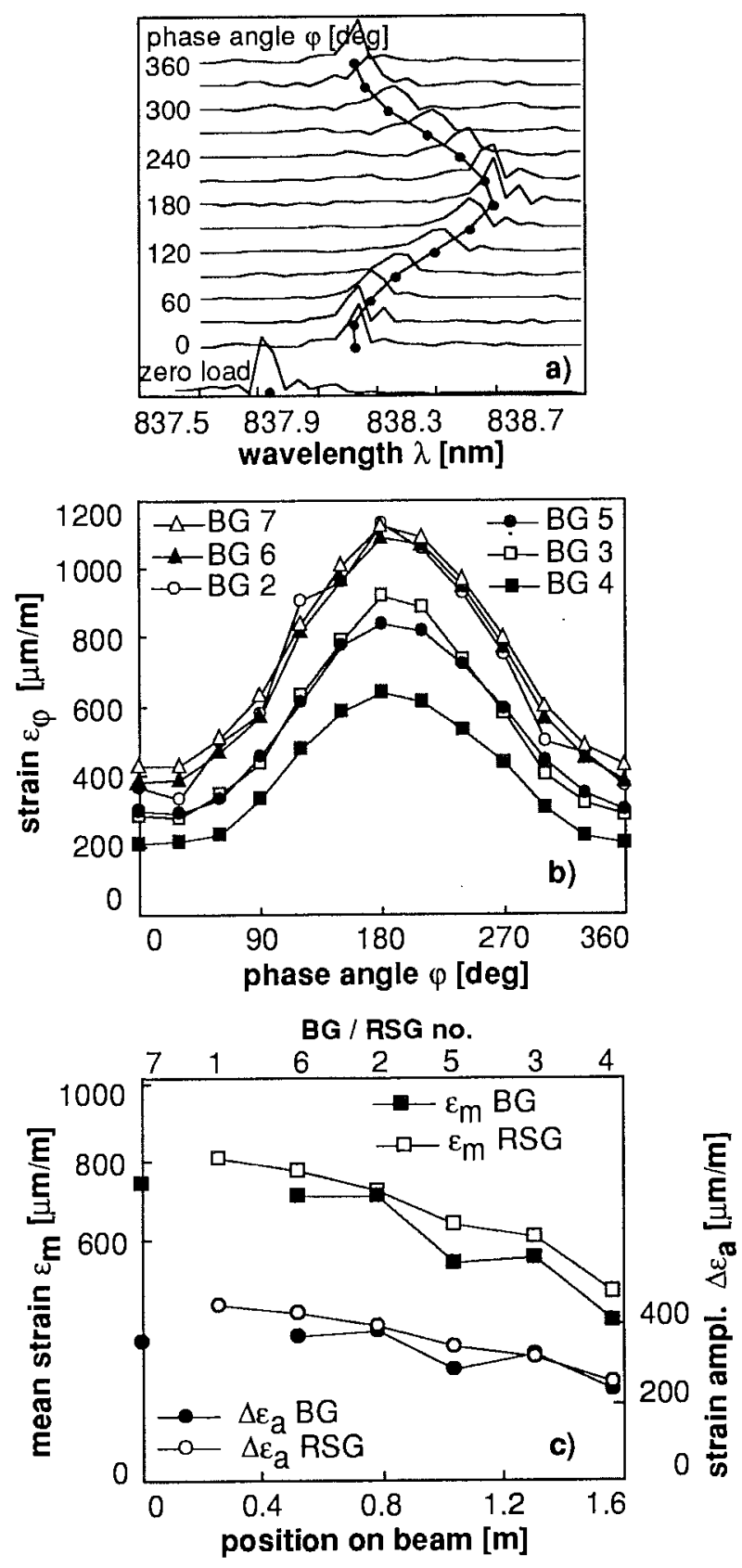

Fig. 9 Measurements during oscillation of the concrete beam. Shown are (a) the decomposed spectra and mean Bragg wavelengths $\lambda_{B}(\cdot)$ at phase angles $\varphi$ from 0 to 360 deg for BG $7 ;$ (b) strain measured with $B G$ array versus phase angle $\varphi$; and (c) mean strain $\epsilon_{m}$ and strain amplitude $\Delta \epsilon_{a}$ measured with $B G s$ and compared with values from RSGs.

tude and mean load $\left(\Delta F_{a}=0.17 F_{\max }, F_{m}=0.61 F_{\max }\right)$ is $\Delta F_{a} / F_{m}=0.28$. When the concrete beam was statically loaded, we found a corresponding strain ratio of $\left(\Delta \epsilon_{a} / \epsilon_{m}\right)_{\text {static }} \approx 0.28$. In the dynamic case we found $\left(\Delta \epsilon_{a} / \epsilon_{m}\right)_{\text {dynamic }} \approx 0.5$. This means that the dynamic strain amplitude is 1.8 times higher. The excessive amplitude can be explained by the dynamic properties of the concrete beam, which is an oscillatory system.
The agreement of the measurements with BGs and RSGs is not as good as in the static case. However, considering the lower signal-to-noise ratio due to the decomposition and the lower spectral resolution, the agreement with the RSGs is acceptable. With these results, the potential of the method was demonstrated. Both the signal-to-noise ratio and the spectral resolution can be improved.

\section{Conclusions and Outlook}

We demonstrated the performance of optical fiber Bragg grating arrays fabricated in line on a draw tower by measuring strain distributions. The measured objects were a large concrete beam and a prestressing carbon fiber cable. For the concrete beam, the dependence of the strain field on load was obtained for static and dynamic load cycles. For the prestressing carbon fiber cable, the optical fiber sensors were shown to be much easier to apply than resistance strain gauges. A slight nonlinearity in the load-strain curve caused by the lateral confinement pressure could be confirmed by the optical measurements.

The reflection signals of the Bragg gratings were transmitted with a multimode fiber over a distance of $400 \mathrm{~m}$ and detected with a Michelson interferometer used as Fourier spectrometer. We obtained a strain resolution of 3 to 15 $\mu \mathrm{m} / \mathrm{m}$ for all seven gratings of an array. The measuring method itself could be improved in terms of strain resolution $(<1 \mu \mathrm{m} / \mathrm{m})$ and sampling frequency; however, this was not the intent of this work. The measurements with optical fiber Bragg gratings agreed well with those made simultaneously with electrical resistance strain gauges in close vicinity. Although the measurements were made on two objects out of the laboratory (real measurement conditions), the errors were shown to be less than $\pm(15 \mu \mathrm{m} / \mathrm{m}$ $+0.02 \times \epsilon)$.

We measured at local point and extensometric sensor configurations and observed differences in peak shape and measurement resolution for the two configurations. The extensometric configuration showed about a threefold better performance and a higher accuracy in determining the slope of the load-strain-curve. The peaks of some local point sensors broadened up to nine times their original width.

Oscillation frequency, mean strain, and strain amplitude of an oscillating concrete beam were obtained by analyzing the phase and wavelength of the reflected light of the Bragg gratings. We found the dynamic strain amplitude to be 1.8 times higher than in the static case due to the dynamic properties of the concrete beam.

It has been shown that surface attachment and embedding of optical fiber Bragg gratings can be a competitive method for measuring the distribution of strain in civil engineering. For broad acceptance of this method, reliability, long-term stability, and good fatigue behavior have to be demonstrated.

\section{Acknowledgments}

We would like to express our thanks to P. Anderegg and E. Cattarin, colleagues in the electronics/metrology section at EMPA, for their valuable technical support and to $\mathrm{M}$. Waldvogel for preparing the testing area and machines. Special thanks go to Prof. U. Meier, director of EMPA Dübendorf, for discussing measurement requirements for 
civil engineering; to A. Maissen for providing the carbon fiber cables; and to A. Winistörfer for his help with the vacuum bagging method.

\section{References}

1. A. T. Alavie, R. Maaskant, M. M. Ohm, S. Rizkalla, and R. M. Mea sures, "Application and characterisation of intracore grating sensors in a CFRP prestressed concrete girder." Proc. SPIE 2191, 103-110 (1994)

2. A. Méndez and T. F. Morse, "Overview of optical fiber sensors embedded in concrete," Proc. SPIE 1798, 205-216 (1992)

3. J. D. Prohaska, E. Snitzer, B. Chen, M. H. Maher, E. G. Nawy, and W. W. Morey, "Fiber optic Bragg grating strain sensors in large scale concrete structures," Proc. SPIE 1798, 286-294 (1992).

4. Ph. M. Nellen, H. Pierhöfer, R. Brönnimann, and U. Sennhauser, "Absolute strain measurements with multiplexed low coherence demodulated fiber Fabry-Perot sensors," Proc. SPIE 2360, 518-521 (1994)

5. Ph. M. Nellen, R. Brönnimann, U. Sennhauser, C. G. Askins, and M. A. Putnam, "Applications of distributed fiber Bragg grating sensors in civil engineering," Proc. SPIE 2507, 14-24 (1995).

6. G. Meltz, W. W. Morey, and W. H. Glenn, "Formation of Bragg gratings in optical fibers by a transverse holographic method," Opt. Lett. 14(15), 823-825 (1989).

7. J. S. Sirkis, "Unified approach to phase-strain-temperature models for smart structure interferometric optical fiber sensors, part 1: development, part 2: application," Opt. Eng. 32(4), 752-772 (1993).

8. A. Bertholds and R. Dandliker, "Determination of the individual strain-optic coefficients in single-mode optical fibers," J. Lightwave Technol. 6(1), 17-20 (1988)

9. C. G. Askins, T. E. Tsai, G. M. Williams, M. A. Putnam, M. Bashkansky, and E. J. Friebele, "Fiber Bragg reflectors prepared by a single excimer pulse," Opt. Lett. 17(11), 833 (1992)

10. C. G. Askins, M. A. Putnam, G. M. Williams and E. J. Friebele, "Stepped-wavelength optical-fiber arrays fabricated in line on a draw tower,"' Opt. Lett. 19(2), 147-149 (1994)

11. C. G. Askins, M. A. Putnam, and E. J. Friebele, "Instrumentation for interrogating many-element fiber Bragg grating arrays," Proc. SPIE 2444, 252-266 (1995).

12. M. Deuring, "Post-strengthening of concrete structures with pretensioned advanced composites," Ph.D. thesis, EMPA, summarized in U. Meier, "EMPA research report no. 224," ACM News, 1-2 (1994)

13. A. Maissen and C. A. M. De Smet, "Comparison of concrete beams prestressed with carbon fibre reinforced plastic and steel strands," "Non-metallic (FRP)-reinforcement for concrete structures," in Proc. Second Int. RILEM Symposium (FRPRCS-2), L. Taerwe, Ed. pp 430-439 (1995)

14. R. M. Measures, S. Huang, M. Le Blanc, M. Ohn, and A. T. Alavie, "Bragg intra-grating sensing, implications for smart structures," Proc. SPIE 2191, 436-445 (1994)

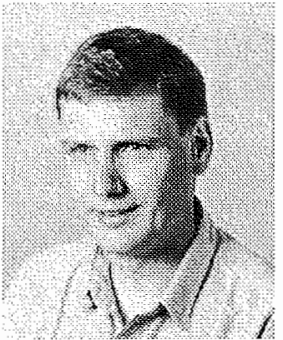

Philipp M. Nellen received his diploma and $P h D$ degrees in physics from the Swiss Federal Institute of Technology in Zürich $(\mathrm{ETHZ})$. He was an assistant researcher and teaching assistant in the optics laboratory at the Institute of Quantum Electronics and worked in the field of integrated optical chemo- and biosensors. In 1993 he joined the Swiss Federal Laboratories for Materials Testing and Research (EMPA). Here his major interest is fiber optical sensors for structural monitoring.

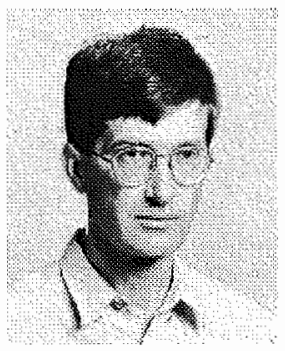

Rolf Brönnimann studied physics at the Swiss Federal Institute of Technology in Zürich (ETHZ). He received his doctorate in natural sciences from the ETHZ. He held a postdoctoral position at Bell Laboratories in Murray Hill, New Jersey, and is now with the Swiss Federal Laboratories for Materials Testing and Research, where he is involved in the application of fiber optical sensors.

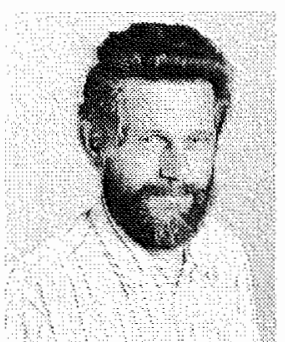

Urs Sennhauser is head of the Section Electronics/Metrology of the Swiss Federal Laboratories for Materials Testing and Research. He received his diploma and his PhD degrees in physics from the Swiss Federal Institute of Technology in Zurich. Strain and displacement measurements for testing performance and reliability of materials and structures are a long-term major topic in the section. Current research activities include fiber optic strain sensors and optical 3-D metrology such as electronic speckle interferometry (ESPI) and moiré techniques.

Charles G. Askins has studied materials properties of optical fibers at the U.S. Naval Research Laboratory in Washington DC since 1981. His current interests include UV photosensitivity in Ge-doped silica fibers and demodulation techniques for use with fiber Bragg gratings used in sensing

Martin A. Putnam: Biography and photograph not available. 
